\title{
Forest Fire Detection System using Wireless Sensor Networks and Machine Learning
}

\section{Udaya Dampage ( $\nabla$ udayadmpage@ieee.org )}

General Sir John Kotelawala Defence University

D. M. L. N. Bandaranayake

General Sir John Kotelawala Defence University

W. M.R. S. Wanasinghe

General Sir John Kotelawala Defence University

K. O. Kottahachchi

General Sir John Kotelawala Defence University

W. A. B. Jayasanka

General Sir John Kotelawala Defence University

\section{Research Article}

Keywords: Forest fire, Wireless Sensor Networks, Machine Learning, Low latency, Low power consumption

Posted Date: July 21st, 2021

DOI: https://doi.org/10.21203/rs.3.rs-722627/v1

License: (c) (1) This work is licensed under a Creative Commons Attribution 4.0 International License. Read Full License

Version of Record: A version of this preprint was published at Scientific Reports on January 7th, 2022. See the published version at https://doi.org/10.1038/s41598-021-03882-9. 


\title{
Forest Fire Detection System using Wireless Sensor Networks and Machine Learning
}

\author{
Udaya Dampage *1, D. M. L. N. Bandaranayake ${ }^{2}$, W. M.R. S. Wanasinghe ${ }^{3}$, K. O. \\ Kottahachchi $^{4}$, and W. A. B. Jayasanka 5 \\ ${ }^{1-5}$ Electrical, Electronic and Telecommunication Engineering, General Sir John Kotelawala Defence University, Ratmalana 10390, Sri \\ Lanka. \\ *Corresponding author: Udaya Dampage (e-mail: udayadampage@ieee.org, dampage@kdu.ac.lk). \\ ORCID ID: 0000-0003-0151-8218
}

\begin{abstract}
Forest fires have become a major threat around the world, causing many negative impacts on human beings and forest ecosystems. Even though rapid climatic changes arising from high environmental pollution, greenhouse effects, etc. have caused this situation, a higher percentage of forest fires occur due to human activities. Therefore, to minimize the destruction caused by forest fires, the need to detect forest fires at their initial stage is needed. This paper proposes a model that can be used to detect forest fires at the initial stage using a wireless sensor network. Furthermore, to acquire more accurate fire detection, a machine learning regression model is proposed. Moreover, thorough attention is given to sensor node design and node placement in the forest to be fitted in the forest environment to minimize the damage and harmful effects caused by wild animals, weather conditions, etc. to the system.
\end{abstract}

Index Terms - Forest fire, Wireless Sensor Networks, Machine Learning, Low latency, Low power consumption

\section{Introduction}

13 - orest fires are disasters that cause extensive damage to the entire world in economic, ecological, and environmental ways.

14 These fires can be caused by natural reasons, such as high temperatures that can create a spontaneous combustion of dry fuel

15 such as sawdust, leaves, lightning, etc., or by human activities, such as unextinguished campfires, arson, inappropriately burned debris, etc. [1] According to research, $90 \%$ of the world's forest fire incidents have occurred as a result of the abovementioned human carelessness. [1] The increase in carbon dioxide levels in the atmosphere due to forest fires contributes to the greenhouse effect and climate change. Additionally, ash destroys much of the nutrients in the soil and can cause erosion, which may result in floods and landslides.

In earlier times, forest fires were detected using watchtowers, which were not efficient because they were based on human observations. In recent history and even the present day, there have been several forest fire detection methods implemented, such as watchtowers, satellite image processing methods, optical sensors, and digital camera-based methods, [2] though there are many drawbacks, such as inefficiency, power consumption, latency, accuracy and implementation costs. To address these drawbacks, a forest fire detection system using wireless sensor networks is proposed in this paper.

Wireless sensor networks (WSNs) are self-configured and infrastructure-free wireless networks that help monitor physical or environmental conditions and pass these data through the network to a designated location or sink where the data can be observed and analysed. [3] Efficiency and low power consumption are the major advantages of a WSN. In the proposed detection system, the wireless sensor nodes are deployed according to cellular architecture to cover the entire area with sensors to monitor temperature, relative humidity, light intensity level, and carbon monoxide (CO) level using microcontroller, transceiver module, and power components. The power supply to the sensor node is provided using batteries as the primary power supply, and solar panels are used as the secondary power supply. These sensor nodes are specially designed with a spherical shape to withstand damage caused by environmental conditions as well as animals.

The sensor readings for each parameter are checked with a preset threshold ratio and a ratio that is calculated continuously in the node in real time, and only the ratios that exceed the preset ratio are sent from the sensor node to the base station for further analytical processing. The network utilized for this transmission is in the architecture of tree topology considering facts such as low power consumption, reduced latency, less complexity, etc. Cluster heads are used in this network to gather data from several sensor nodes and pass them on to the base station or the gateway node. The gateway node is an interface that connects the network with the secondary analysis process.

For the analysis process, a machine learning regression model was used along with threshold ratio analysis to enhance detection accuracy. For the training and testing process of the model, data were collected during fire and nonfire situations in different areas and under different climatic conditions. During the data collection process, 7000 data samples were collected, where a data sample 
included temperature, relative humidity, light intensity level, and CO level at a particular time. Eighty percent of the collected data were randomly used as training data for the model, and the remaining $20 \%$ were used as test data.

If the outcome of the machine learning model indicates a fire in a specific area, a text message will be sent to the mobile phone numbers of the authorized officers in responsible units. As this process is designed with a minimum delay, the fire can be detected within the initial stage, and the responsible parties can take necessary actions in a shorter period, which will minimize the damage.

\section{Related Work}

Forest fire detection has been a focus of many researchers for the last decade because of increased forest fire case reports from all over the world due to severe damage to society and the environment. Many methods have been proposed to detect forest fires, such as camera-based systems, WSN-based systems, and machine learning application-based systems, with both positive and negative aspects and performance figures of detection. Due to the higher probability of accurate and early detection due to the use of multiple sensor sources and deployment of sensor nodes in areas not visible to satellites, wireless sensor networks have a more positive outlook, and they have become the more applicable technology in many fields. [4].

Many researchers have focused on environmental parameters, such as air temperature, relative humidity, barometric pressure, sound, light intensity, soil moisture, and wind speed and direction, along with gases, such as $\mathrm{CO}, \mathrm{CO}_{2}$, methane, $\mathrm{H}_{2}$, and hydrocarbons apart from smoke, to detect forest fire conditions by considering the variations in these parameters during a fire [5] [6], and sensors have been selected according to range, sensitivity, power consumption, and cost. [7] [8]

As supplying power to a sensor node is a challenging task in forested areas, utilizing only battery options is difficult because they do not last long, and distributing power using a wire would require a higher cost to deploy in a large forest. Therefore, many researchers have proposed solar-powered systems as secondary power sources along with rechargeable batteries as the main power source [4] [6], while some researchers have proposed solar batteries because they last longer [9]. To reduce the power consumption of sensor nodes, techniques such as keeping selected components active while others are deactivated have been proposed. [10] [11] [12]

Most WSN-based detection systems are centred around a base station due to the memory and processing limitations of the nodes. Important and partially processed data are transferred to the base station through wireless media for processing and enabling relevant actions, while the base station also acts as the gateway between the sensor nodes and the system user. [4] [9]

When constructing a WSN, communicating data among the relevant entities is the main objective, and star topology and mesh topology-based networks have been proposed in many papers because of the different attributes in their systems. A mesh topology was chosen over a star topology because of its ability to self-organize, self-configure, and automatically establish among nodes in a network. [13]. As a smaller number of nodes involved for transmission results in minimum energy consumption, concepts based on cluster heads have been used[14]. To minimize the loss of energy and data packages during transfer, a cluster-tree network topology structure was proposed[15].

To place sensor nodes in the most effective configuration to detect fire conditions, a sensor node was proposed at three different heights to perform different parameter measurements, while some authors have suggested covering sensor nodes to avoid direct sunlight exposure and minimize the false alarm rate. [4] [10] As the network connectivity of service providers in forest areas is not robust, communication techniques that use dedicated network paths such as LoRa, ZigBee, and XBee have been used as the communication infrastructure. When considering attributes such as transmission range and other relevant configurations, most papers have suggested using the LoRa module for transmission. [13]

Most papers have suggested having threshold value-based fire detection on a sensor node, and if the exceeded threshold has remained the same, then a sink determines the location and will send an alarm to the fire department[11]. Because of the environmental parameter variations according to the place and time, threshold values are configured by the user considering geographic situations, climatic changes, seasonal changes, etc. after sensors obtain the data from the surrounding. [10].

A fusion information process was proposed, where information from multiple sources is considered in making the final decision, which is better than using those sources individually, and two algorithms based on the threshold ratio method and Dempster-Shafer theory were used. [4] To enhance the detection accuracy, machine learning applications have been proposed in many papers based on different machine learning approaches, such as support vector machine (SVM) classification [14] and regression techniques, such as logistic regression. However, applying machine learning techniques to fire detection systems has many limitations, such as the limited amount of energy, the energy required for data processing, the short range of communication and limited computations, the complexity of ML algorithms when executing on sensor nodes and the difficulty of being distributed on every sensor node. [16] [17]

\section{Materials and Methods}




\section{Sensor Node Design}

The sensor node was designed with a spherical shape to withstand external forces and with characteristics to prevent damage due to the harsh conditions prevailing in tropical forests. The sensor node is used to detect the temperature, humidity, light intensity level, and CO level, which are detected from the lower side of the node facing downwards (Fig. 12). This is for the protection of the sensors from harmful effects of environmental conditions such as rain, strong winds, and objects such as leaves. The mounting brackets of the node on the tree trunk and supporters are connected on the rear side of the sensor node (Fig. 10).

Sensor Node Deployment

After conducting a number of tests, the maximum sensing range of a sensor node was determined to be five metres. After conducting a site survey of the average foliage height, the sensor node was mounted on a tree trunk one metre above the ground to conduct detection tests of initial stage surface fire conditions effectively. The deployment of the sensor nodes in a forest was carried out after a similar site survey of the average foliage height and as per the cellular architecture (Fig. 3) to cover the whole area of interest. As the maximum sensing range of a sensor node is five metres, a sensor node can cover a five-metre radius.
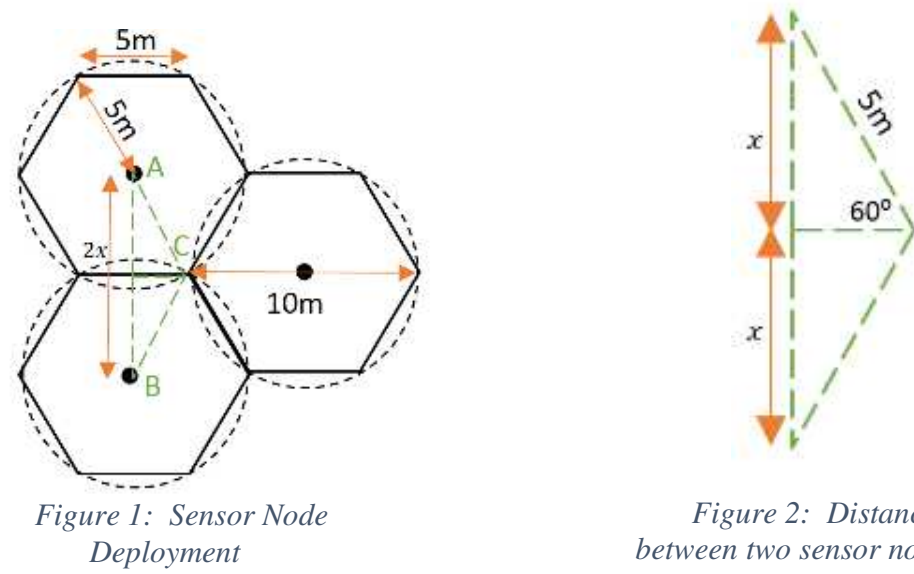

Figure 2: Distance between two sensor nodes

According to Fig. 2-3, the distance between two sensor nodes is calculated as follows.

$$
\begin{gathered}
x=5 m \times \sin (60)=4.33 m \\
2 x=4.33 m \times 2=8.66 m
\end{gathered}
$$

For the data transmission from the sensor node to the gateway node (Fig. 3), an nrf24L01 module was utilized, which has a maximum transmission range of $100 \mathrm{~m}$ in a forest-type environment. To collect data from sensor nodes beyond $100 \mathrm{~m}$ and for ease of communication, the sensor nodes are arranged into clusters. Each cluster has a cluster head that collects data from sensor nodes and transmits them to the gateway node. Deployment of the cluster heads is also arranged according to the cellular architecture, where a single cluster head covers an area of a circle with a $50 \mathrm{~m}$ radius when considering the maximum range of the transceiver module. As per the calculations, the distance between two cluster heads is $86.66 \mathrm{~m}$, and each cluster head collects data from 100 sensor nodes. The set of data values sent by the sensor nodes on the condition of exceeding the threshold ratio are collected by the relevant cluster heads and passed to the gateway for further analysis. A cluster head deployed beyond $100 \mathrm{~m}$ from the gateway node can pass data through intermediate cluster heads. 


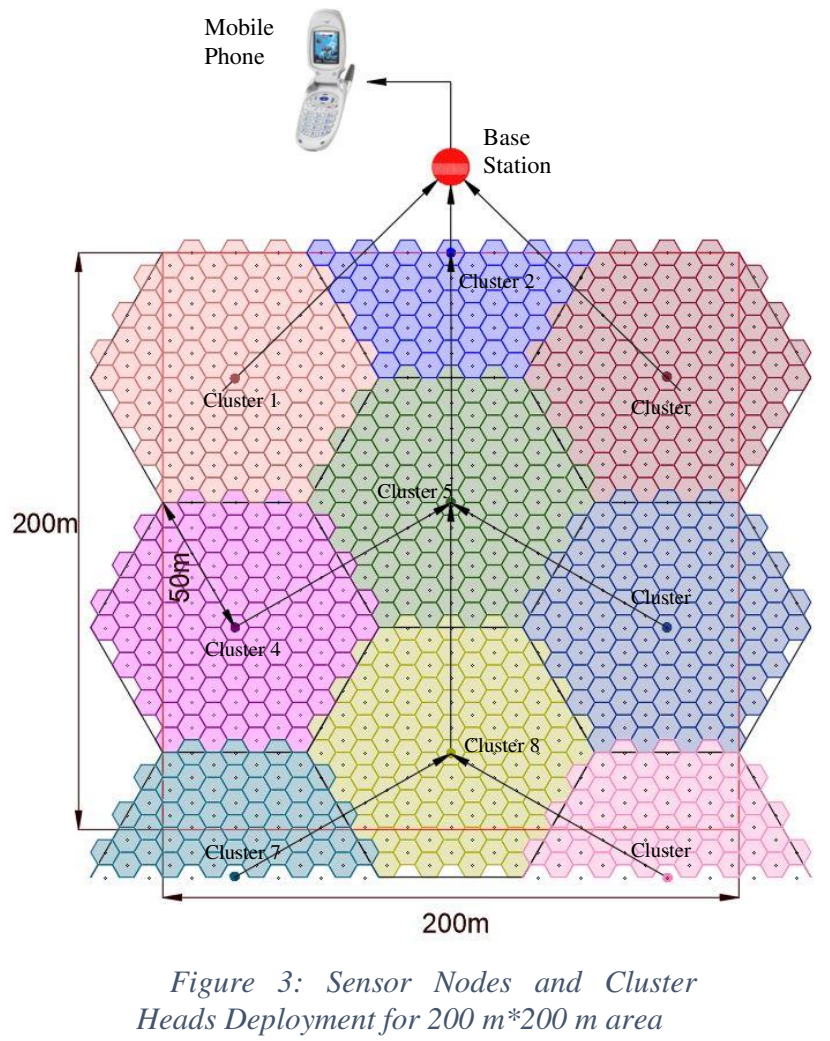

151

152

153

154

155

156

157

158

159

160

161

162

163

164

165

166

167

168

169

170

171

172

173

\section{Results and Discussion}

\section{Data Collection}

For the detection of fire conditions, two analytical methods were used, namely, threshold ratio analysis and analysis using a machine learning algorithm. To carry out these analytical processes, data were collected by creating several controlled fire conditions. The aforementioned conditions were created in an area of $1 \mathrm{~m}^{2}$, and the sensor node was mounted on a post one metre above the ground and placed one metre from the fire. The data collection was carried out in different climatic zones during the morning, afternoon, and night hours.

\section{Threshold Ratio Analysis}

The environmental parameters, including temperature, relative humidity, light intensity level, and CO level, were monitored by the system under different climatic conditions in different climatic zones during the morning, afternoon, and night hours. The threshold ratio $R_{T H}$ was determined during these extensive trials. A ratio was calculated continuously within the sensor node by reading each parameter $R$ by the respective sensors with thirty second periods. If the calculated ratio of a single parameter exceeds the threshold ratio value three consecutive times, then a set of ten data values was sent to the gateway node from each parameter. To determine the threshold ratio, data were collected at the different areas and different times of the day, as presented in Fig. 6 to Fig. 9.

$$
\text { Threshold Ratio }=\frac{\text { Current data value }}{\text { Data value before } 30 \mathrm{~s}}
$$

$$
\text { Threshold Ratio }\left(R_{T H}\right)=\frac{d_{t}}{d_{t-30}} \quad \text { Eqn. (1) }
$$




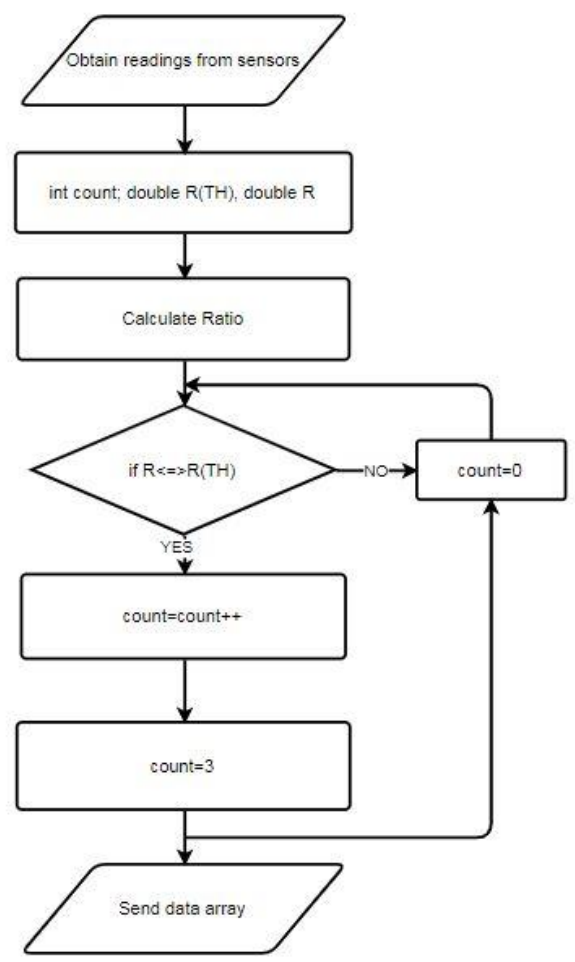

Figure 4: The decision flow of the sensor node

174
The decision flow of the sensor node is depicted in Fig. 4. When the temperature and light intensity are checked, if the condition is $R T H<=R$, in regard to the humidity and CO level, the condition should be $R T H>=R$.

Machine Learning Algorithm

To minimize the effect of spontaneous or random errors and erroneous sensor readings, a long-term pattern analysis was carried out by a machine learning algorithm.

A dataset of 7000 samples was collated where a single data sample comprised values of temperature, relative humidity, light intensity level, and $\mathrm{CO}$ level at a particular instance within a certain climatic zone. These data samples were collected by monitoring no fire situations as well as fire situations that were created in the different climatic zones during the morning, afternoon, and night hours. The K-means technique was used to separate the data samples into two clusters: fire and no fire, as plotted in Fig. 5.

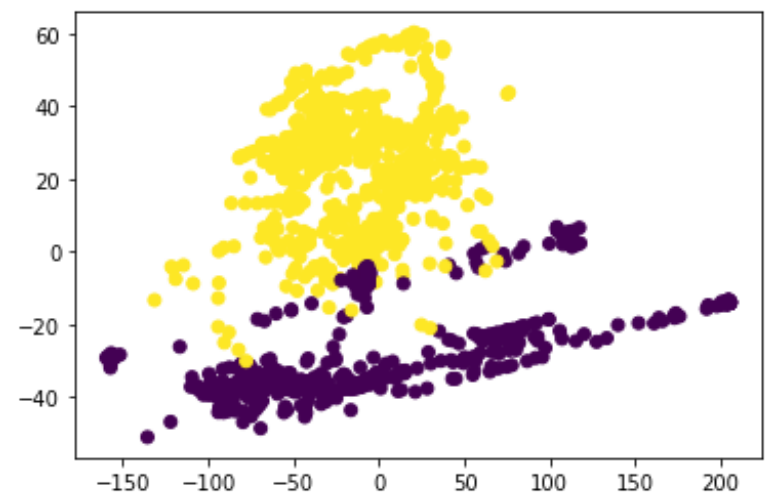

Figure 5: Clustered data samples using K-means method

Eighty percent of the prepared dataset was used for the training process of the model, and the remaining $20 \%$ was used for the testing process. The dataset was trained by using a multiple linear regression model. 
190

191

192

193

194

195

196

197

198

199

200

201

202

203

204

205

206

207

208

209

210

211

212

213

214

215

216

217

The data sent from sensor nodes after threshold analysis are collected at the gateway node and fed into the machine learning model. If a fire situation is detected after an analysis, the model provides a result as a fire situation along with the area where it has occurred. Then, the responsible authorities are informed about the fire by a text message to respective mobile phone numbers.

\section{Field Testing}

The initial testing of the system was performed at land adjoining the Kanneliya Forest Reserve [6.183120723327043, 80.4165345965101], Sri Lanka, and the other was at land adjoining the Knuckles Mountain Range Wildlife Reserve [7.45857419273649, 80.77990606784306], Sri Lanka, which are often subjected to frequent forest fires. A controlled fire was created, and sensor nodes were placed to detect the fire. One cluster head and the base node were implemented for the transmission of data and further analysis. In both locations, the tests were performed in the morning, afternoon, and night to check the system applicability to different times of day.

\section{Results}

For a performance analysis of the sensor node, the predefined threshold ratio values were determined for each parameter, namely, temperature, relative humidity, light intensity level, and carbon monoxide level. To determine the threshold ratio values for all four parameters, data values were obtained by creating 15 controlled fires, and values were determined for fire situations created at different climatic zones during the morning, afternoon, and night hours. The graphs of Fig. 6 to Fig. 9 show each parameter's ratio $v$ s time, where the ratio was obtained by calculating the ratio between the current reading and the reading before 30 seconds as per Eq. (1). The readings that were used to plot the graphs below commenced 60 seconds before the time instant when the fire was started.

Three possible threshold ratios were selected for all four parameters considering the variation profile of each parameter, and each fire situation was analysed with the selected threshold ratio values. For temperature and light intensity, 1.15, 1.1, and 1.05 were selected, as those parameter ratios should be higher than 1 , while for humidity and CO levels, $0.85,0.8$, and 0.95 were selected, as those ratios should be less than 1 in fire situations, as presented in Table 1.

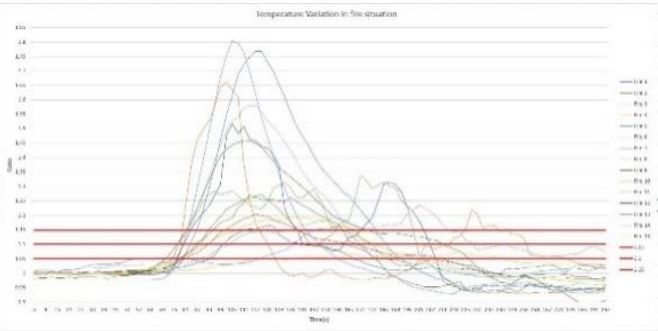

Figure 6: Temperature ratio vs Time

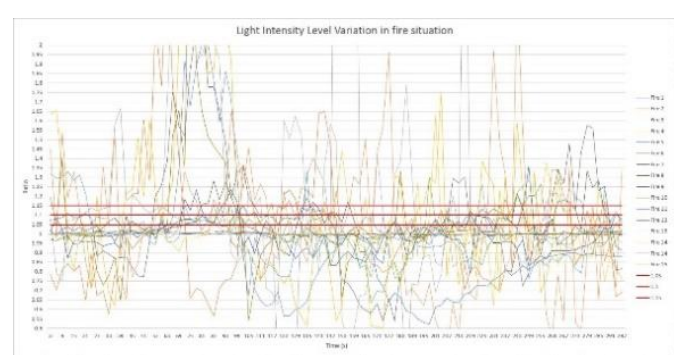

Figure 8: Light Intensity Ratio vs Time

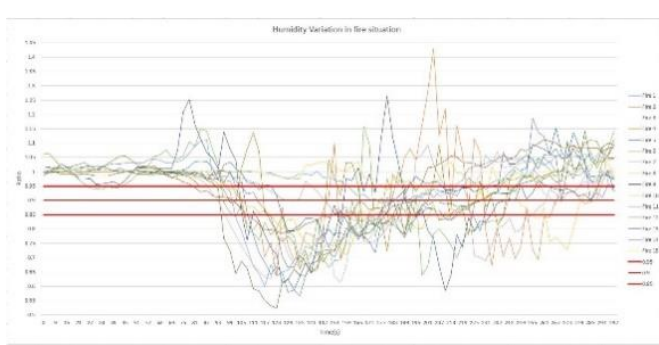

Figure 7: Humidity Ratio vs Time

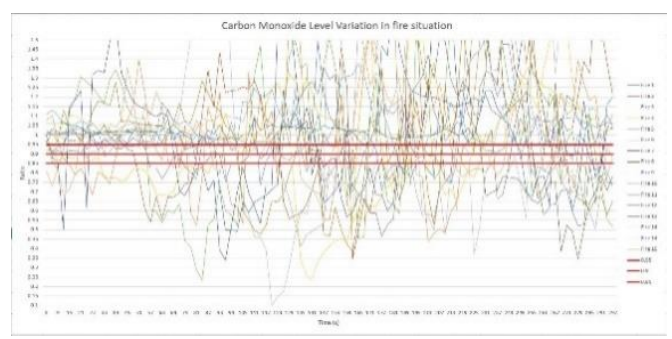

Figure 9: CO Level Ratio vs Time 
Table 1: Decided Threshold Ratio Value for Each Parameter

\begin{tabular}{|l|c|}
\hline Parameter & Threshold Ratio Value \\
\hline Temperature & 1.05 \\
\hline Relative humidity & 0.95 \\
\hline Light intensity level & 1.15 \\
\hline CO level & 0.85 \\
\hline
\end{tabular}

The sensor node was designed in a spherical shape to be stable and damage resistant. When designing the node, the sensors and other equipment placement in the node extensively consider mounting ability and safety, as depicted in Fig. 10 to Fig. 12.

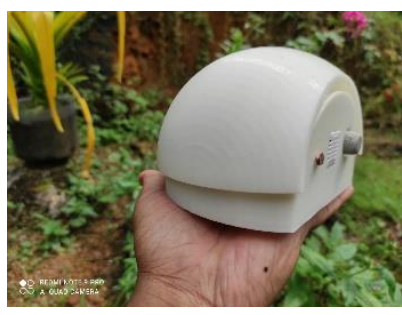

Figure 10: Spherical exterior design

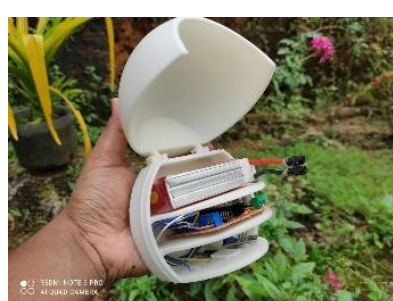

Figure 11: Interior component placement

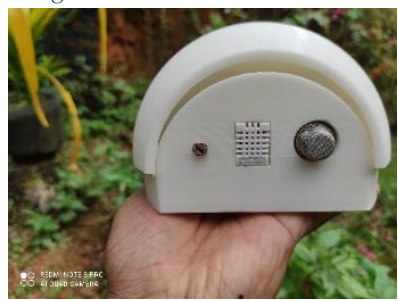

Figure 12: Sensors placed at the downside

After performing a number of tests, the $5 \mathrm{~m}$ range was observed as the effective area that a single sensor node can cover. The variation in average delay $v s$ height was observed, as depicted in Fig. 13; hence, the height of the mounting point from the ground level was determined to be $1 \mathrm{~m}$.

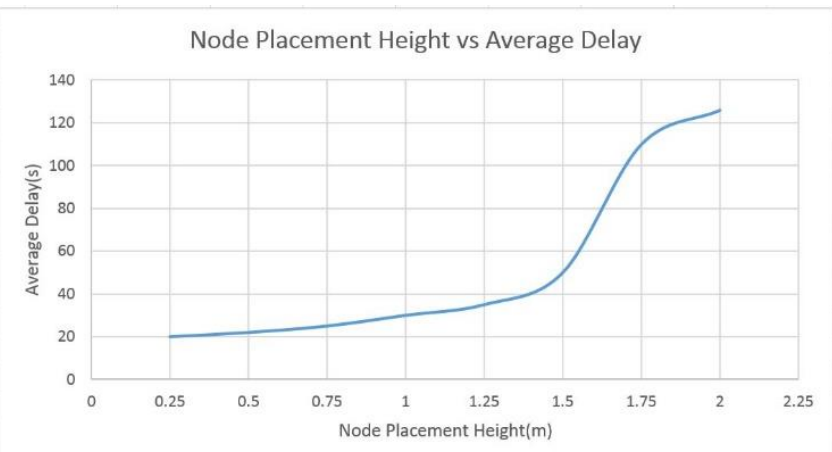

Figure 13: Node Placement Height vs Average Delay 
On training the machine learning regression model using $80 \%$ of the dataset with 7000 samples and testing it by the remaining $20 \%$, the theoretical accuracy of the final output obtained was $81 \%$. After testing the system for twenty-two fire scenarios and twenty-eight no-fire scenarios, node accuracy, practical accuracy of the machine learning model, and overall system accuracy were calculated, and the data are presented in Table 2 and Fig. 14.

Table 2: Accuracy Graph of System, Node and Machine Learning Model

\begin{tabular}{|c|l|c|c|c|c|c|}
\hline & \multicolumn{1}{|c|}{ Scenario } & $\begin{array}{c}\text { Total } \\
\text { Instances }\end{array}$ & $\begin{array}{c}\text { Accurate } \\
\text { Instances }\end{array}$ & $\begin{array}{c}\text { Error } \\
\text { Instances }\end{array}$ & Accuracy & Error \\
\hline \multirow{3}{*}{ Node } & Fire & 22 & 22 & 0 & $100 \%$ & $0 \%$ \\
\cline { 2 - 7 } & No Fire & 28 & 18 & 10 & $64.28 \%$ & $35.72 \%$ \\
\cline { 2 - 7 } & Overall & 50 & 40 & 10 & $80 \%$ & $20 \%$ \\
\hline \multirow{3}{*}{ MIL } & Fire & 22 & 19 & 3 & $86.36 \%$ & $13.64 \%$ \\
\cline { 2 - 7 } & No Fire & 16 & 14 & 2 & $87.50 \%$ & $12.50 \%$ \\
\cline { 2 - 7 } & Overall & 38 & 33 & 5 & $86.84 \%$ & $13.15 \%$ \\
\hline \multirow{3}{*}{ Overall } & Fire & 22 & 19 & 3 & $86.36 \%$ & $13.64 \%$ \\
\cline { 2 - 7 } & No Fire & 28 & 26 & 2 & $92.85 \%$ & $7.15 \%$ \\
\cline { 2 - 7 } & Overall & 50 & 45 & 5 & $90 \%$ & $10 \%$ \\
\hline
\end{tabular}

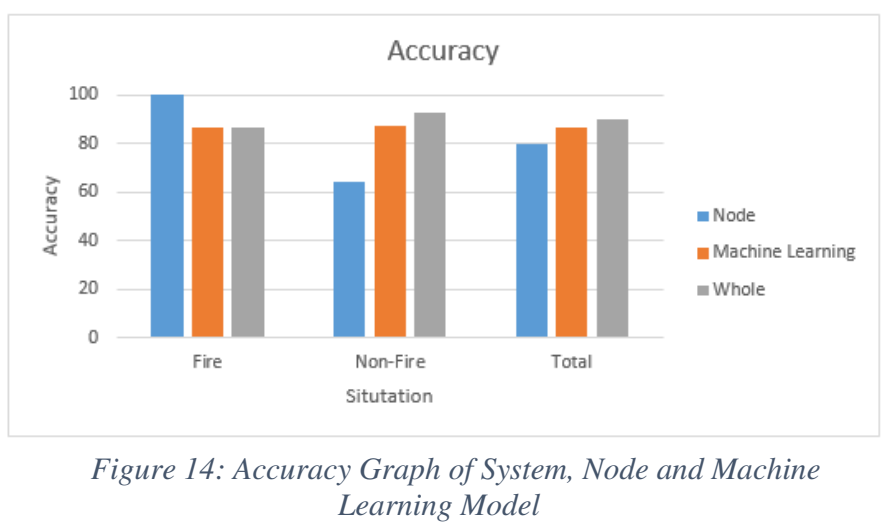

A statistical t-test was performed to determine the significance of the parameters that were utilized to detect fire conditions. The test was conducted for all four parameters: temperature, relative humidity, light intensity level, and carbon monoxide level considering fire and no fire situations. The probability values obtained for all four parameters were less than the critical value $(\propto$ $=0.05$ ) as indicated in Table 3 . In advance, the probability values were less than 0.001 , which implies that those parameters have a strong correlation with the condition of fire. Therefore, it can be concluded that the above parameters can be well utilized for the detection of fire conditions. [18]

Table 3: Significance Level for Each Parameter

\begin{tabular}{|l|c|}
\hline Parameter & $\begin{array}{c}\text { Significance Level (p- } \\
\text { value) }\end{array}$ \\
\hline Temperature & $1.77 \times 10^{-44}$ \\
\hline Relative Humidity & $1.03 \times 10^{-45}$ \\
\hline Light Intensity Level & $1.73 \times 10^{-6}$ \\
\hline CO Level & $8.16 \times 10^{-12}$ \\
\hline
\end{tabular}

\section{Conclusion}

The proposed system for forest fire detection using wireless sensor networks and machine learning is an effective method for fire detection in forests that provides more accurate results. Here, to obtain a more accurate output, an analysis takes place in both the sensor node and base station. For the system, to fit in any weather condition, climatic condition, or area, a threshold ratio is introduced for analysis in the sensor node. In the case of node deployment, it can be mounted at any place in the forest even if there is no preinstalled network connectivity, as the transceiver module is based on a dedicated network structure that can be selfbuilt. Because of the primary power supply provided by rechargeable batteries and a secondary solar power supply, a solution for 
available power is also provided. Alerting the relevant authorities through the text message completes the process of the proposed system with lower latency.

\section{Data Availability}

The datasets generated and/or analysed during the current study are available from the corresponding author on reasonable request.

\section{References}

[1] R. Nelson, "untamedscience.com," April 2019. [Online]. Available: https://untamedscience.com/blog/theenvironmental-impact-of-forest-fires/. [Accessed 30 December 2020].

[2] A. A. A. Alkhatib, "A Review on Forest Fire Detection Techniques," International Journal of Distributed Sensor Networks, 2014.

[3] M. M. I. M. A. Matin, Overview if Wireless Sensor Network, Intech Open, 2012.

[4] L. A. T. J. A. A. J. A. A. Arnoldo Díaz-Ramíreza, "Wireless Sensor Networks and Fusion Information Methods," in The 2012 Iberoamerican Conference on Electronics Engineering and Computer Science, México, 2012.

[5] D. C.-F. A. A. J. A. A. R. Antonio Molina-Pico, "Forest Monitoring and Wildland Early Fire Detection by a Hierarchical Wireless Sensor Network," Journal of Sensors, pp. 1-8, 2016.

[6] R. A. A. S. I. H. E. George Emil Sakr, "Forest fire detection wireless sensor node," in Advances in Forest Fire Research, Imprensa da Universidade de Coimbra, 2014, pp. 1395-1406.

[7] F. F. D. a. P. U. N. w. IoT, "Pantech Solutions," 4 September 2018. [Online]. Available: https://www.pantechsolutions.net/forest-fire-detection-and-prediction-using-nodemcu-withiot\#product_tabs_description_tabbed. [Accessed 13 March 2020].

[8] Y. L. H. X. K. L. T. YongMin Liu, "Forest fire monitoring, detection and decision making systems by wireless sensor network," in 2018 Chinese Control And Decision Conference (CCDC), Shenyang, 2018.

[9] Y. G. G. C. Y. J. J. L. Yongsheng Liu, "A Novel Accurate Forest Fire Detection System Using Wireless Sensor Networks," in 2011 Seventh International Conference on Mobile Ad-hoc and Sensor Networks, Japan, 2011.

[10] A. a. A. D. a. M.-M. N. a. C. B. a. C. S. Bayo, "Early Detection and Monitoring of Forest Fire with a Wireless Sensor Network System," in Procedia Engineering, Spain, 2010.

[11] A. A. Alkhatib, “Wireless Sensor Network for Forest Fire Detection and Decision Making," International Journal of Advances in Engineering Science and Technology, vol. volume 2, pp. 299-310, November 2013.

[12] S. B. S. M. A. C. a. I. K. Sakib Abdullah, "A Wireless Sensor Network for Early Forest Fire Detection and Monitoring as a Decision Factor in the Context of a Complex Integrated Emergency Response System," in 2017 IEEE Workshop on Environmental, Energy, and Structural Monitoring Systems (EESMS), Milan, Italy, 2017.

[13] Adnan, A. E. U. Salam, A. Arifin and M. Rizal, "Forest Fire Detection Using LoRa Wireless Mesh Topology," in 2018 2nd East Indonesia Conference on Computer and Information Technology (EIConCIT), Makassar, Indonesia, 2018.

[14] S. S. U. C. a. C. G. Y. Singh, "Distributed Event Detection in Wireless Sensor Networks for Forest Fires," in 2013 UKSim 15th International Conference on Computer Modelling and Simulation, Cambridge, 2013.

[15] E. A. Kadir, S. L. Rosa and A. Yulianti, "Application of WSNs for Detection Land and Forest Fire in Riau Province Indonesia," in 2018 International Conference on Electrical Engineering and Computer Science (ICECOS), Pangkal Pinang, Indonesia, 2018.

[16] Y. S. N. K. V. M. Aditi Kansal, "Detection of Forest Fires using Machine Learning Technique: A Perspective," in Third International Conference on Image Infonnation Processing , India, 2014.

[17] T. Zhang, Q. Zhao and Y. Nakamoto, "Faulty Sensor Data Detection in Wireless Sensor Networks Using Logistical Regression," in 2017 IEEE 37th International Conference on Distributed Computing Systems Workshops (ICDCSW), Atlanta, 2017.

[18] J. Fernandez, "towards datascience," 11 April 2020. [Online]. Available: https://towardsdatascience.com/thestatistical-analysis-t-test-explained-for-beginners-and-experts-fd0e358bbb62. [Accessed 10 February 2021]. 